\title{
On self-reciprocal functions in two variables
}

\author{
by R. U. Verma (Lucknow)
}

1. Introduction. Agarwal [1] derived the general form of self-reciprocal functions and two theorems of kernels connecting self-reciprocal functions of different orders, though Gray (1931) and Erdelyi (1936) have given some examples of self-reciprocal functions involving two complex variables. Erdelyi has employed Tricomi's theorem for two variables in the Operational Calculus in his researches. Reed (1944) has proved certain theorems on Mellin transform of functions of two variables. The main results of Reed are embodied in the following theorems:

THEOREM I. If in the strip $a<{ }^{\circ} \sigma<\beta, a<r<b$,

(i) the function $f(r, s)$ of two complex variables is regular,

(ii) the integral

$$
\int_{-\infty}^{+\infty} \int_{-\infty}^{+\infty}|f(\sigma+i t, r+i \tau)| d t d \tau
$$

is absolutely convergent,

(iii) $|f(r, s)| \rightarrow 0$ as $t$ and $\tau$ approach infinity independently.

If for positive $x$ and $y$

$$
g(x, y)=\frac{1}{(2 \pi i)^{2}} \int_{\sigma-i \infty}^{\sigma+i \infty} \int_{r-i \infty}^{r+i \infty} x^{-r} y^{-s} f(r, s) d r d s,
$$

then

$$
f(r, s)=\int_{0}^{\infty} \int_{0}^{\infty} x^{r-1} y^{s-1} g(x, y) d x d y
$$

THEOREM II. If

(i) $x$ and $y$ are positive,

(ii) $g(x, y)$ is piecewise continuous,

(iii) $\int_{0}^{\infty} \int_{0}^{\infty} x^{\sigma-1} y^{\gamma-1} g(x, y) d x d y$ is absolutely convergent between the strips $\alpha<\sigma<\beta, a<r<b$, then the converse of Theorem I is true.

The object of this paper is to generalize an integral transform and its extension to two variables, and to develop the theory of self-reciprocal 
functions in two variables on the basis of Reed's theory of double Mellin transform.

2. A generalized Hankel transform is defined by the relation [4]

$$
\left.=\int_{0}^{\infty} G(x) \mid \begin{array}{l}
k-m-\frac{1}{2}-v / 2,-k+m+\frac{1}{2}+v / 2 \\
v / 2-\lambda-m, v / 2-\lambda+m,-v / 2+\lambda+m,-v / 2+\lambda-m
\end{array}\right) f(y) d y,
$$

where $m$ is not an integer or zero.

Generalized Hankel transforms introduced by Bhise [2] is a particular case of (2.1) when $\lambda=-m$. Thus in turn it includes all the transforms as special case which are particular cases of the transform due to Bhise.

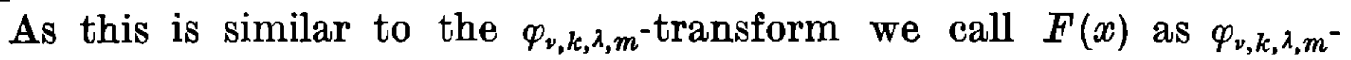
transform of $f(x)$. If $f(x)=F(x)$, then $f(x)$ shall be called self-reciprocal in $\varphi_{\nu, k, \lambda, m}$-transform and we shall say that $f(x)$ is $R_{\nu}(k, \lambda, m)$.

3. A function $f(x, y)$ of two complex variables $x$ and $y$ will be said to be self-reciprocal in the generalized Hankel transform of order $\left(v, \nu^{\prime}\right)$ if it satisfied the integral equation

(3.1) $f(x, y)$

$$
\begin{aligned}
& =\int_{0}^{\infty} \int_{0}^{\infty} G\left(x u \mid \begin{array}{l}
k-m-\frac{1}{2}-v / 2,-k+m+\frac{1}{2}+v / 2 \\
v / 2-\lambda-m, v / 2-\lambda+m,-v / 2+\lambda+m,-v / 2+\lambda-m
\end{array}\right) \times \\
& \underset{2,4}{2,1}\left(y u^{\prime} \mid \begin{array}{l}
k^{\prime}-m^{\prime}-\frac{1}{2}-v^{\prime} / 2,-k^{\prime}+m^{\prime}+\frac{1}{2}+v^{\prime} / 2 \\
v^{\prime} / 2-\lambda^{\prime}-m^{\prime}, v^{\prime} / 2-\lambda^{\prime}+m^{\prime},-v^{\prime} / 2+\lambda^{\prime}+m^{\prime},-v^{\prime} / 2+\lambda^{\prime}-m^{\prime}
\end{array}\right) \times \\
& \times f\left(u, u^{\prime}\right) d u d u^{\prime} \text {. }
\end{aligned}
$$

Drawing the analogy from the case of one variable we shall denote such a function by the symbol $R_{v ; v^{\prime}}\left(k, \lambda, m ; k^{\prime}, \lambda^{\prime}, m^{\prime}\right)$.

If $M(r, s)$ denotes the double Mellin transform of $f(x, y)$, then we have

$$
M(r, s)=\int_{0}^{\infty} \int_{0}^{\infty} f(x, y) x^{r-1} y^{s-1} d x d y
$$

Substituting the expression for $f(x, y)$ in (3.2) from (3.1) and changing the order of integration, we $\operatorname{get}\left({ }^{1}\right)$

$$
\begin{gathered}
M(r, s)=\frac{\Gamma\left(\frac{3}{2}-k+m+v / 2-r\right) \Gamma_{\times}(v / 2-\lambda \pm m+r)}{\Gamma\left(v / 2-k+m+\frac{1}{2}+r\right) \Gamma_{\times}(v / 2+1+r-\lambda \pm m)} \times \\
\times \frac{\Gamma\left(\frac{3}{2}-k^{\prime}+m^{\prime}+v^{\prime} / 2-s\right) \Gamma_{\times}\left(v^{\prime} / 2-\lambda^{\prime} \pm m^{\prime}+s\right)}{\Gamma\left(\nu^{\prime} / 2-k^{\prime}+m^{\prime}+\frac{1}{2}+s\right) \Gamma_{\times}\left(v^{\prime} / 2+1-s-\lambda^{\prime} \pm m^{\prime}\right)} M(1-r, 1-s),
\end{gathered}
$$

it being assumed that (3.1) is absolutely convergent.

(1) For the sake of brevity we denote $\Gamma(a+b) \Gamma(a-b)$ by $\Gamma_{\times}(a \pm b)$. 


\section{Hence}

$$
\begin{aligned}
f(x, y)=\frac{1}{(2 \pi i)^{2}} & \int_{c-i \infty}^{c+i \infty} \int_{i^{\prime}-i \infty}^{c^{\prime}+i \infty} \frac{\Gamma_{\times}(v / 2-\lambda \pm m+r)}{\Gamma\left(\frac{1}{2}-k+m+v / 2+r\right)} \times \\
& \times \frac{\Gamma_{\times}\left(\nu^{\prime} / 2-\lambda^{\prime} \pm m^{\prime}+s\right)}{\Gamma\left(\frac{1}{2}-k^{\prime}+m^{\prime}+\nu^{\prime} / 2+s\right)} \psi(r, s) x^{-r} y^{-s} d r d s,
\end{aligned}
$$

where

$$
\psi(r, s)=M(r, s) \frac{\Gamma\left(\frac{1}{2}-k+m+v / 2+r\right) \Gamma\left(\frac{1}{2}-k^{\prime}+m^{\prime}+v^{\prime} / 2+s\right)}{\Gamma_{\times}(v / 2+r-\lambda \pm m) \Gamma_{\times}\left(v^{\prime} / 2+s-\lambda \pm m^{\prime}\right)}
$$

and satisfies the functional equation.

$$
\psi(r, s)=\psi(1-r, 1-s) .
$$

It remains now to justify the various steps in the above analysis.

Assuming the restrictions imposed on $M(r, s)$ and $f(x, y)$ in the Reed's theorem cited above to hold, we find that for the change in the order of integration of the double integrals to be valid it is sufficient, if only

(a) $x, y$ - integrals will be absolutely convergent, when $R(\nu-2 \lambda+$ $+2 m+2 r)>0, R(v-2 \lambda-2 m+2 r)>0, R\left(v / 2+r-k+m+\frac{1}{2}\right)>0$. And similar conditions with $v=v^{\prime}, m=m^{\prime}, \lambda=\lambda^{\prime}, k=k^{\prime}, r=s$.

(b) $u, u^{\prime}$ - integrals will be absolutely convergent, when

(i) $f\left(u, u^{\prime}\right)=O\left(u^{\xi}, u^{\prime \eta}\right)$ for small values of $u$ and $u^{\prime}$,

(ii) $f\left(u, u^{\prime}\right)=O\left(\frac{1}{u}, \frac{1}{u^{\prime}}\right)$ as $u$ and $u^{\prime} \rightarrow \infty$,

(iii) $R(\nu / 2+\xi-\lambda \pm m)>0$ and $m$ is not an integer or zero.

And similar conditions with $\nu=\nu^{\prime}, m=m^{\prime}, \lambda=\lambda^{\prime}, k=k^{\prime}, \xi=\eta$ and $r=s$.

The existence of relation (3.4) demands that $|M(r, s)| \rightarrow 0$ as $t$ and $\tau$ tend to infinity independently, where $r=\sigma+i t, s=r+i \tau$.

This implies from (3.5) that

$$
\left|\frac{\Gamma_{\times}(\nu / 2-\lambda \pm m+r) \Gamma_{\times}\left(\nu^{\prime} / 2-\lambda^{\prime} \pm m^{\prime}+s\right)}{\Gamma\left(\frac{1}{2}-k+m+\nu / 2+r\right) \Gamma\left(\frac{1}{2}-k^{\prime}+m^{\prime}+\nu^{\prime} / 2+s\right)} \psi(r, s)\right| \rightarrow 0
$$

as $t$ and $\tau \rightarrow \infty$.

Using asymptotic expansion for the Gamma function, we get

$$
\psi(r, s)=O\left(e^{(\lambda / 2-a+\eta)[|t|+|\tau|]}\right)
$$

for every positive $\eta$, however, small $0<a \leqslant \pi$. 


\section{References}

[1] R. P. Agarwal, On self-reciprocal functions involving two complex variables, Ganita 1 (1950), p. 17.25.

[2] V. M. Bhise, Operators of fractional integration and a generalized Henkel transform, Collect. Math. 16 (1964), p. 201.

[3] I. S. Reed, The Mellin type of Double integral, Duke Math. Journ. 11 (1944).

[4] R. U. Verma, Thesis for Ph. D. degree (1968) from Lucknow University (India).

DEPARTMENT OF MATHFMATICS, LUCKNOW UNIVERSITY

Lucknow (India) 